\title{
Lower and Upper Solutions Method for Positive Solutions of Fractional Boundary Value Problems
}

\author{
R. Darzi, ${ }^{1}$ B. Mohammadzadeh, ${ }^{2}$ A. Neamaty, ${ }^{3}$ and D. Bǎleanu ${ }^{4,5,6}$ \\ ${ }^{1}$ Department of Mathematics, Neka Branch, Islamic Azad University, P.O. Box 48411-86114, Neka, Iran \\ ${ }^{2}$ Department of Mathematics, Sari Branch, Islamic Azad University, P.O. Box 48161-19318, Sari, Iran \\ ${ }^{3}$ Department of Mathematics, University of Mazandaran, P.O. Box 47416-95447, Babolsar, Iran \\ ${ }^{4}$ Department of Mathematics and Computer Science, Faculty of Art and Sciences, Cankaya University, Yenimahalle, \\ 06810 Ankara, Turkey \\ ${ }^{5}$ Department of Chemical and Materials Engineering, Faculty of Engineering, King Abdulaziz University, P.O. Box 80204, \\ Jeddah 21589, Saudi Arabia \\ ${ }^{6}$ Institute of Space Sciences, P.O. Box, MG-23, R 76900 Magurele, Bucharest, Romania
}

Correspondence should be addressed to R. Darzi; r.drazi@yahoo.com

Received 10 May 2013; Revised 11 July 2013; Accepted 1 August 2013

Academic Editor: Juan J. Trujillo

Copyright (C) 2013 R. Darzi et al. This is an open access article distributed under the Creative Commons Attribution License, which permits unrestricted use, distribution, and reproduction in any medium, provided the original work is properly cited.

We apply the lower and upper solutions method and fixed-point theorems to prove the existence of positive solution to fractional boundary value problem $D_{0^{+}}^{\alpha} u(t)+f(t, u(t))=0,0<t<1,2<\alpha \leq 3, u(0)=u^{\prime}(0)=0, D_{0^{+}}^{\alpha-1} u(1)=\beta u(\xi), 0<\xi<1$, where $D_{0^{+}}^{\alpha}$ denotes Riemann-Liouville fractional derivative, $\beta$ is positive real number, $\beta \xi^{\alpha-1} \geq 2 \Gamma(\alpha)$, and $f$ is continuous on $[0,1] \times[0, \infty)$. As an application, one example is given to illustrate the main result.

\section{Introduction}

In the recent years, fractional calculus has been one of the most interesting issues that have attracted many scientists, especially in the fields of mathematics and engineering sciences. Many natural phenomena can be presented by boundary value problems of fractional differential equations. Many authors in different fields such as chemical physics, fluid flows, electrical networks, and viscoelasticity try to present a model of these phenomena by boundary value problems of fractional differential equations [1-4]. In order to achieve extra information in fractional calculus, interested readers can refer to more valuable books that are written by other authors [5-20].

The existence and multiplicity of solutions or positive solutions of nonlinear fractional differential equation (FDE) by the use of fixed point theorems, Leray-Shauder theory, and so forth are mentioned in some papers $[6,8,12,20,21]$. Few papers have considered the boundary value problems of fractional differential equations $[12,14]$. By the use of some fixed point theorems on cones, Zhang [15] obtained the existence of positive solution for the equation

$$
D_{0^{+}}^{\alpha} u(t)+f(t, u(t))=0, \quad 0<t<1,1<\alpha \leq 2,
$$

with the boundary conditions

$$
u(0)=u(1)=0 .
$$

In [22], Liang and Zhang applied lower and upper solutions method and fixed point theorems to obtain some results on the existence of positive solutions for the following BVPs:

$$
\begin{gathered}
D_{0^{+}}^{\alpha} u(t)=f(t, u(t)), \quad 0<t<1,3<\alpha \leq 4, \\
u(0)=u(1)=u^{\prime}(0)=u^{\prime}(1)=0,
\end{gathered}
$$

where $D^{\alpha}$ denotes Riemann-Liouville fractional derivative. 
In this paper, we investigate the existence of positive solution for a nonlocal BVP of FDE,

$$
\begin{gathered}
D_{0^{+}}^{\alpha} u(t)+f(t, u(t))=0, \quad 0<t<1,2<\alpha \leq 3, \\
u(0)=u^{\prime}(0)=0, \quad D_{0^{+}}^{\alpha-1} u(1)=\beta u(\xi),
\end{gathered}
$$

using lower and upper solutions method and fixed point theorem, where $D^{\alpha}$ denotes standard Riemann-Liouville fractional derivative, $\beta \xi^{\alpha-1} \geq 2 \Gamma(\alpha)$, and $f \in C([0,1] \times$ $[0, \infty),[0, \infty))$.

The main result of this paper can be seen in Theorem 10 . In Theorem 10, we use the following conditions:

$\left(\mathrm{H}_{1}\right) f(t, u(t)) \in C\left([0,1] \times[0, \infty), \mathbb{R}^{+}\right)$is nondecreasing with respect to $u$,

$\left(\mathrm{H}_{2}\right) f(t, \varrho(t)) \neq 0$ for $t \in(0,1)$,

$\left(\mathrm{H}_{3}\right)$ there exist a positive constant $\lambda<1$ such that $k^{\lambda} f(t, u) \leq f(t, k u)$, for all $0 \leq k \leq 1$, and the Schauder fixed-point theorem to show that problem (4)-(5) has a positive solution.

\section{Basic Definitions and Preliminaries}

In this section, we present the necessary definitions and lemmas that will be used to prove our new results.

Definition 1 (see $[5,6]$ ). The Riemann-Liouville fractional integral of order $\alpha>0$ of a function $f:(0, \infty) \rightarrow \mathbb{R}$ is defined by

$$
I_{0^{+}}^{\alpha} f(t)=\frac{1}{\Gamma(\alpha)} \int_{0}^{t}(t-s)^{\alpha-1} f(s) d s, \quad n-1<\alpha \leq n,
$$

provided that the right-hand side is pointwise defined on $(0, \infty)$.

Definition 2 (see $[5,6]$ ). The Riemann-Liouville fractional derivative of order $\alpha>0$ of a function $f:(0, \infty) \rightarrow \mathbb{R}$ is defined by

$$
\begin{array}{r}
D_{0^{+}}^{\alpha} f(t)=\frac{1}{\Gamma(n-\alpha)} \frac{d^{n}}{d x^{n}} \int_{0}^{t}(t-s)^{\alpha-1} f(s) d s, \\
n-1<\alpha \leq n,
\end{array}
$$

where $n=[\alpha]+1$, provided that the right-hand side is pointwise defined on $(0, \infty)$.

Definition 3 (see $[5,6])$. A function $\mu(t) \in C^{2}[0,1]$ is called a lower solution of problem (4)-(5) if $\mu(t)$ satisfies

$$
\begin{gathered}
-D_{0^{+}}^{\alpha} \mu(t) \leq f(t, \mu(t)), \quad 0<t<1,2<\alpha \leq 3, \\
\mu(0) \leq 0, \quad \mu^{\prime}(0) \leq 0, \quad D_{0^{+}}^{\alpha} \mu(t) \leq \beta \mu(\xi) .
\end{gathered}
$$

Definition 4 (see $[7,8]$ ). A function $\mu(t) \in C^{2}[0,1]$ is called an upper solution of problem (4)-(5) if $\mu(t)$ satisfies

$$
\begin{gathered}
-D_{0^{+}}^{\alpha} \mu(t) \geq f(t, \mu(t)), \quad 0<t<1,2<\alpha \leq 3, \\
\mu(0) \geq 0, \quad \mu^{\prime}(0) \geq 0, \quad D_{0^{+}}^{\alpha} \mu(t) \geq \beta \mu(\xi) .
\end{gathered}
$$

Lemma 5 (see $[7,8]$ ). Let $u \in C(0,1) \cap L^{1}(0,1)$. Then the fractional differential equation

$$
D^{\alpha} u(t)=0
$$

has

$$
\begin{gathered}
u(t)=c_{1} t^{\alpha-1}+c_{2} t^{\alpha-2}+\cdots+c_{n} t^{\alpha-n}, \\
\text { for some } c_{i} \in \mathbb{R}, i=1, \ldots, n,
\end{gathered}
$$

as a unique solution.

Lemma 6 (see $[7,8]$ ). Let $u \in C(0,1) \cap L^{1}(0,1)$ with a fractional derivative of order $\alpha>0, n-1<\alpha \leq n(n \in \mathbb{N})$, that belongs to $C(0,1) \cap L(0,1)$. Then

$$
\begin{gathered}
I_{0^{+}}^{\alpha} D_{0^{+}}^{\alpha} u(t)=u(t)+c_{1} t^{\alpha-1}+c_{2} t^{\alpha-2}+\cdots+c_{n} t^{\alpha-n}, \\
\text { for some } c_{i} \in \mathbb{R}, i=1, \ldots, n .
\end{gathered}
$$

Lemma 7. If $\beta \xi^{\alpha-1} \geq 2 \Gamma(\alpha)$, then for $0 \leq y(t) \in C[0,1]$, the problem,

$$
\begin{gathered}
D_{0^{+}}^{\alpha} u(t)+y(t)=0, \quad 0<t<1,2<\alpha \leq 3, \\
u(0)=u^{\prime}(0)=0, \quad D_{0^{+}}^{\alpha-1} u(1)=\beta u(\xi),
\end{gathered}
$$

has a unique positive solution

$$
\begin{aligned}
u(t)= & -\frac{1}{\Gamma(\alpha)} \int_{0}^{t}(t-s)^{\alpha-1} y(s) d s \\
& +\frac{1}{\beta \xi^{\alpha-1}-\Gamma(\alpha)} \int_{0}^{1} t^{\alpha-1} y(s) d s \\
& +\frac{\beta}{\left[\beta \xi^{\alpha-1}-\Gamma(\alpha)\right] \Gamma(\alpha)} \int_{0}^{\xi} t^{\alpha-1}(\xi-s)^{\alpha-1} y(s) d s .
\end{aligned}
$$

Proof. We can apply Lemma 6 to reduce (13) to an equivalent integral equation

$$
u(t)=-\frac{1}{\Gamma(\alpha)} \int_{0}^{t}(t-s)^{\alpha-1} y(s) d s+c_{1} t^{\alpha-1}+c_{2} t^{\alpha-2}+c_{3} t^{\alpha-3}
$$

for some $c_{1}, c_{2}, c_{3} \in \mathbb{R}$. From $u(0)=0$ and $u^{\prime}(0)=0$ in (14), we have $c_{2}=c_{3}=0$. On the other hand, $D^{\alpha-1} u(1)=\beta u(\xi)$ yields

$$
\begin{aligned}
c_{1}= & \frac{1}{\beta \xi^{\alpha-1}-\Gamma(\alpha)} \int_{0}^{t} y(s) d s \\
& +\frac{\beta}{\left[\beta \xi^{\alpha-1}-\Gamma(\alpha)\right] \Gamma(\alpha)} \int_{0}^{\xi}(\xi-s)^{\alpha-1} y(s) d s .
\end{aligned}
$$

Then, the unique solution of problem is given by $u(t)$. Obviously, $u(t) \geq 0$ if $y(t) \geq 0$ on $t \in[0,1]$. The proof is complete. 


\section{Main Result}

In this section, we present and prove our main result.

Lemma 8. Suppose that $\beta \xi^{\alpha-1} \geq 2 \Gamma(\alpha)$. Given that $y \in$ $C[0,1]$, the Green function for the problem (13)-(14) is given by

$$
\begin{aligned}
& G(t, s) \\
& \quad\left\{\begin{array}{cl}
\left(-(t-s)^{\alpha-1}\left[\beta \xi^{\alpha-1}-\Gamma(\alpha)\right]\right. & \\
\left.+\Gamma(\alpha) t^{\alpha-1}+\beta t^{\alpha-1}(\xi-s)^{\alpha-1}\right) & \\
\times\left(\left[\beta \xi^{\alpha-1}-\Gamma(\alpha)\right] \Gamma(\alpha)\right)^{-1}, & 0 \leq s \leq t \leq 1, s \leq \xi, \\
\left(-(t-s)^{\alpha-1}\left[\beta \xi^{\alpha-1}-\Gamma(\alpha)\right]\right. & \\
\left.+\Gamma(\alpha) t^{\alpha-1}\right) & \\
\times\left(\left[\beta \xi^{\alpha-1}-\Gamma(\alpha)\right] \Gamma(\alpha)\right)^{-1}, & 0 \leq \xi \leq s \leq t \leq 1, \\
\frac{\Gamma(\alpha) t^{\alpha-1}+\beta t^{\alpha-1}(\xi-s)^{\alpha-1}}{\left[\beta \xi^{\alpha-1}-\Gamma(\alpha)\right] \Gamma(\alpha)}, & 0 \leq t \leq s \leq \xi \leq 1, \\
\frac{t^{\alpha-1}}{\beta \xi^{\alpha-1}-\Gamma(\alpha)}, & t \leq s, s \geq \xi .
\end{array}\right.
\end{aligned}
$$

Proof. By Lemma 7, for $t \leq \xi$, we have

$u(t)$

$$
\begin{aligned}
&=-\frac{1}{\Gamma(\alpha)} \int_{0}^{t}(t-s)^{\alpha-1} y(s) d s+\frac{1}{\beta \xi^{\alpha-1}-\Gamma(\alpha)} \\
& \times\left[\left(\int_{0}^{t}+\int_{t}^{\xi}+\int_{\xi}^{1}\right) t^{\alpha-1} y(s) d s\right] \\
&+\frac{\beta}{\left[\beta \xi^{\alpha-1}-\Gamma(\alpha)\right] \Gamma(\alpha)} \\
& \times\left[\left(\int_{0}^{t}+\int_{t}^{\xi}\right) t^{\alpha-1}(\xi-s)^{\alpha-1} y(s) d s\right] \\
&= \int_{0}^{t}\left(\left(-(t-s)^{\alpha-1}\left[\beta \xi^{\alpha-1}-\Gamma(\alpha)\right]\right.\right. \\
&+\left.+\Gamma(\alpha) t^{\alpha-1}+\beta t^{\alpha-1}(\xi-s)^{\alpha-1}\right) \\
&+\int_{t}^{\xi} \frac{\Gamma(\alpha) t^{\alpha-1}+\beta t^{\alpha-1}(\xi-s)^{\alpha-1}}{\left[\beta \xi^{\alpha-1}-\Gamma(\alpha)\right] \Gamma(\alpha)} y(s) d s \\
&+\int_{\xi}^{1} \frac{\Gamma(\alpha) t^{\alpha-1}}{\left[\beta \xi^{\alpha-1}-\Gamma(\alpha)\right] \Gamma(\alpha)} y(s) d s \\
&=\int_{0}^{1} G(t, s) y(s) d s .
\end{aligned}
$$

For $t \geq \xi$, we have

$u(t)$

$$
\begin{gathered}
=-\frac{1}{\Gamma(\alpha)}\left[\left(\int_{0}^{\xi} \int_{\xi}^{t}\right)\right](t-s)^{\alpha-1} y(s) d s+\frac{1}{\beta \xi^{\alpha-1}-\Gamma(\alpha)} \\
\times\left[\left(\int_{0}^{\xi}+\int_{\xi}^{t}+\int_{t}^{1}\right) t^{\alpha-1} y(s) d s\right] \\
+\frac{\beta}{\left[\beta \xi^{\alpha-1}-\Gamma(\alpha)\right] \Gamma(\alpha)} \int_{0}^{\xi} t^{\alpha-1}(\xi-s)^{\alpha-1} y(s) d s \\
=\int_{0}^{\xi}\left(\left(-(t-s)^{\alpha-1}\left[\beta \xi^{\alpha-1}-\Gamma(\alpha)\right]\right.\right. \\
\left.+\Gamma(\alpha) t^{\alpha-1}+\beta t^{\alpha-1}(\xi-s)^{\alpha-1}\right) \\
\left.\quad \times\left(\left[\beta \xi^{\alpha-1}-\Gamma(\alpha)\right] \Gamma(\alpha)\right)^{-1}\right) y(s) d s \\
+\int_{\xi}^{t}\left(\left(-(t-s)^{\alpha-1}\left[\beta \xi^{\alpha-1}-\Gamma(\alpha)\right]+\Gamma(\alpha) t^{\alpha-1}\right)\right. \\
\quad+\int_{t}^{1} \frac{\Gamma(\alpha) t^{\alpha-1}}{\left[\beta \xi^{\alpha-1}-\Gamma(\alpha)\right] \Gamma(\alpha)} y(s) d s \\
=\int_{0}^{1} G(t, s) y(s) d s .
\end{gathered}
$$

The proof is complete.

Lemma 9. Suppose that $u(t) \in C^{2}[0,1]$ and is a positive solution of (4)-(5). Then

$$
m \varrho(t) \leq u(t) \leq M \varrho(t),
$$

where

$$
\varrho(t)=\frac{1}{c \Gamma(\alpha+1)}\left[\beta \xi^{\alpha}-\Gamma(\alpha+1)\right] t^{\alpha-1}-c t^{\alpha},
$$

where $c=\beta \xi^{\alpha-1}-\Gamma(\alpha), \beta \xi^{\alpha-1} \neq \Gamma(\alpha)$, and $m$ and $M$ are two constants.

Proof. Since $u(t) \in C^{2}[0,1]$, there exists $T>0$ so that $|u(t)| \leq$ $T$ for $t \in[0,1]$. We define

$$
\begin{aligned}
m & :=\min _{(t, u) \in[0,1] \times[0, T]} f(t, u(t)), \\
M & :=\max _{(t, u) \in[0,1] \times[0, T]} f(t, u(t)) .
\end{aligned}
$$

Therefore, we have

$$
m \int_{0}^{1} G(t, s) d s \leq \int_{0}^{1} G(t, s) f(s, u(s)) d s \leq M \int_{0}^{1} G(t, s) d s .
$$


On the other hand, by direct computation, we get

$$
\begin{aligned}
\int_{0}^{1} G(t, s) d s \\
=\frac{1}{\left[\beta \xi^{\alpha-1}-\Gamma(\alpha)\right] \Gamma(\alpha+1)} \\
\quad \times\left(\left[\beta \xi^{\alpha-1}-\Gamma(\alpha+1)\right] t^{\alpha-1}-\left[\beta \xi^{\alpha-1}-\Gamma(\alpha)\right] t^{\alpha}\right) .
\end{aligned}
$$

This completes the proof of the lemma.

Theorem 10. The fractional boundary value problem (4)-(5) has a positive solution $u(t)$ if the conditions $\left(H_{1}\right)-\left(H_{3}\right)$ are satisfied.

Proof. Suppose that

$$
\begin{aligned}
& \alpha_{1}=\min \left\{1, \inf _{t \in[0,1]} f(t, \varrho(t))\right\}, \\
& \alpha_{2}=\max \left\{1, \sup _{t \in[0,1]} f(t, \varrho(t))\right\}, \\
& 0<k_{1} \leq \min \left\{\frac{1}{\alpha_{2}},\left(\alpha_{1}\right)^{\lambda /(1-\lambda)}\right\}, \\
& k_{2} \geq \max \left\{\frac{1}{\alpha_{2}},\left(\alpha_{1}\right)^{\lambda /(1-\lambda)}\right\},
\end{aligned}
$$

and $h(t)=\int_{0}^{1} G(t, s) f(s, \varrho(s)) d s$. We show that $\mu(t)=k_{1} h(t)$ and $\nu(t)=k_{2} h(t)$ are lower and upper solutions of (4)-(5), respectively. From Lemma $7, h(t)$ is a positive solution of the following problem:

$$
\begin{gathered}
-D_{0^{+}}^{\alpha} u(t) \leq f(t, \varrho(t)), \quad 0<t<1,2<\alpha \leq 3, \\
u(0)=u^{\prime}(0)=0, \quad D_{0^{+}}^{\alpha-1} u(1)=\beta u(\xi) .
\end{gathered}
$$

We know that $\alpha_{1} \varrho(t) \leq h(t) \leq \alpha_{2} \varrho(t)$. Now, using the assumption of the theorem, we get

$$
\begin{gathered}
k_{1} \alpha_{1} \leq \frac{h(t)}{\varrho(t)} \leq k_{2} \alpha_{2}, \quad \frac{1}{k_{1} \alpha_{2}} \leq \frac{\varrho(t)}{h(t)} \leq \frac{1}{k_{2} \alpha_{1}} \leq 1, \\
\left(k_{1} \alpha_{1}\right)^{\lambda} \geq k_{1}, \quad\left(k_{2} \alpha_{2}\right)^{\lambda} \geq k_{2} .
\end{gathered}
$$

Therefore, from $\left(\mathrm{H}_{3}\right)$ and since $\left(k_{1} \alpha_{1}\right)^{\lambda} \geq k_{1}$, the following relations satisfy

$$
\begin{aligned}
f(t, \mu(t)) & =f\left(t, \frac{\mu(t)}{\varrho(t)} \varrho(t)\right) \geq\left(\frac{\mu(t)}{\varrho(t)}\right)^{\lambda} f(t, \varrho(t)) \\
& \geq\left(k_{1} \alpha_{1}\right)^{\lambda} f(t, \varrho(t))>k_{1} f(t, \varrho(t)), \\
k_{2} f(t, \varrho(t)) & =f\left(t, \frac{\varrho(t)}{\nu(t)} v(t)\right) \geq k_{2}\left(\frac{\varrho(t)}{\nu(t)}\right)^{\lambda} f(t, v(t)) \\
& \geq k_{2}\left(k_{1} \alpha_{1}\right)^{-\lambda} f(t, v(t))>f(t, v(t)) .
\end{aligned}
$$

Consequently

$$
\begin{gathered}
-D_{0^{+}}^{\alpha} \mu(t)=k_{1} f(t, \varrho(t)) \leq f(t, \mu(t)), \\
0<t<1,2<\alpha \leq 3, \\
-D_{0^{+}}^{\alpha} \nu(t)=k_{2} f(t, \varrho(t)) \geq f(t, \nu(t)), \\
0<t<1,2<\alpha \leq 3 .
\end{gathered}
$$

Since $\mu(t)=k_{1} h(t)$ and $\nu(t)=k_{2} h(t)$ satisfy the boundary conditions, $\mu(t)$ and $\nu(t)$ are lower and upper solutions of (4)(5), respectively. Now, we suppose that

$$
g(t, u(t))= \begin{cases}f(t, \mu(t)), & u(t) \leq \mu(t) \\ f(t, u(t)), & \mu(t) \leq u(t) \leq v(t) \\ f(t, v(t)), & u(t) \leq v(t)\end{cases}
$$

and prove that FBVP,

$$
\begin{aligned}
& -D_{0^{+}}^{\alpha} u(t)=g(t, u(t)), \quad 0<t<1,2<\alpha \leq 3, \\
& u(0)=u^{\prime}(0)=0, \quad D_{0^{+}}^{\alpha-1} u(1)=\beta u(\xi),
\end{aligned}
$$

has a solution. Consider operator $T: C^{2}[0,1] \rightarrow C^{2}[0,1]$, with $T u(t)=\int_{0}^{1} G(t, s) g(s, u(s)) d s$, where $G(t, s)$ is defined as in Lemma 8. It is easy to see that $T$ is continuous in $C^{2}[0,1]$. Since $f$ is nondecreasing in $u$ (from $\left(\mathrm{H}_{1}\right)$ ), for $u \in C^{2}[0,1]$, we have

$$
f(t, \mu(t)) \leq g(t, u(t)) \leq f(t, v(t)), \quad t \in[0,1] .
$$

So, there exists a positive constant $M$, such that $|g(t, u(t))| \leq$ $M$. We will show that the operator $T$ is equicontinuous.

Case 1. If $s \leq \xi$,

$$
\begin{aligned}
& \left|T u\left(t_{1}\right)-T u\left(t_{2}\right)\right| \\
& \leq\left|\int_{0}^{1}\left[G\left(t_{2}, s\right)-G\left(t_{1}, s\right)\right] g(s, u(s)) d s\right| \\
& =\mid \int_{0}^{t_{2}} \frac{-\left(t_{2}-s\right)^{\alpha-1}}{\Gamma(\alpha)} g(s, u(s)) d s \\
& \quad+\frac{\Gamma(\alpha) t_{2}^{\alpha-1}}{\left[\beta \xi^{\alpha-1}-\Gamma(\alpha)\right] \Gamma(\alpha)} \int_{0}^{t_{2}} g(s, u(s)) d s \\
& \quad+\frac{\beta t_{2}^{\alpha-1}}{\left[\beta \xi^{\alpha-1}-\Gamma(\alpha)\right] \Gamma(\alpha)} \int_{0}^{t_{2}}(\xi-s)^{\alpha-1} g(s, u(s)) d s \\
& \quad+\frac{\Gamma(\alpha) t_{2}^{\alpha-1}}{\left[\beta \xi^{\alpha-1}-\Gamma(\alpha)\right] \Gamma(\alpha)} \times \int_{t_{2}}^{1} g(s, u(s)) d s
\end{aligned}
$$


Abstract and Applied Analysis

5

$$
\begin{aligned}
& +\frac{\beta t_{2}^{\alpha-1}}{\left[\beta \xi^{\alpha-1}-\Gamma(\alpha)\right] \Gamma(\alpha)} \int_{t_{2}}^{1}(\xi-s)^{\alpha-1} g(s, u(s)) d s \\
& -\int_{0}^{t_{1}} \frac{-\left(t_{2}-s\right)^{\alpha-1}}{\Gamma(\alpha)} g(s, u(s)) d s \\
& -\frac{\Gamma(\alpha) t_{1}^{\alpha-1}}{\left[\beta \xi^{\alpha-1}-\Gamma(\alpha)\right] \Gamma(\alpha)} \int_{0}^{t_{1}} g(s, u(s)) d s \\
& -\frac{\beta t_{1}^{\alpha-1}}{\left[\beta \xi^{\alpha-1}-\Gamma(\alpha)\right] \Gamma(\alpha)} \int_{0}^{t_{1}}(\xi-s)^{\alpha-1} g(s, u(s)) d s \\
& -\frac{\Gamma(\alpha) t_{2}^{\alpha-1}}{\left[\beta \xi^{\alpha-1}-\Gamma(\alpha)\right] \Gamma(\alpha)} \times \int_{t_{1}}^{1} g(s, u(s)) d s \\
& -\frac{\beta t_{1}^{\alpha-1}}{\left[\beta \xi^{\alpha-1}-\Gamma(\alpha)\right] \Gamma(\alpha)} \int_{t_{1}}^{1}(\xi-s)^{\alpha-1} g(s, u(s)) d s \mid \\
& =\mid \int_{0}^{t_{1}}\left[\frac{-\left(t_{2}-s\right)^{\alpha-1}}{\Gamma(\alpha)}+\frac{\left(t_{1}-s\right)^{\alpha-1}}{\Gamma(\alpha)}\right] g(s, u(s)) d s \\
& +\int_{t_{1}}^{t_{2}} \frac{-\left(t_{2}-s\right)^{\alpha-1}}{\Gamma(\alpha)} g(s, u(s)) d s \\
& +\frac{\Gamma(\alpha)\left(t_{2}^{\alpha-1}-t_{1}^{\alpha-1}\right)}{\left[\beta \xi^{\alpha-1}-\Gamma(\alpha)\right] \Gamma(\alpha)} \int_{0}^{t_{1}} g(s, u(s)) d s \\
& +\frac{\Gamma(\alpha) t_{2}^{\alpha-1}}{\left[\beta \xi^{\alpha-1}-\Gamma(\alpha)\right] \Gamma(\alpha)} \int_{t_{1}}^{t_{2}} g(s, u(s)) d s \\
& +\frac{\beta\left(t_{2}^{\alpha-1}-t_{1}^{\alpha-1}\right)}{\left[\beta \xi^{\alpha-1}-\Gamma(\alpha)\right] \Gamma(\alpha)} \int_{0}^{t_{1}}(\xi-s)^{\alpha-1} g(s, u(s)) d s \\
& +\frac{\beta t_{2}^{\alpha-1}}{\left[\beta \xi^{\alpha-1}-\Gamma(\alpha)\right] \Gamma(\alpha)} \times \int_{t_{1}}^{t_{2}}(\xi-s)^{\alpha-1} g(s, u(s)) d s \\
& +\frac{\Gamma(\alpha)\left(t_{2}^{\alpha-1}-t_{1}^{\alpha-1}\right)}{\left[\beta \xi^{\alpha-1}-\Gamma(\alpha)\right] \Gamma(\alpha)} \int_{t_{2}}^{1} g(s, u(s)) d s \\
& -\frac{\Gamma(\alpha) t_{1}^{\alpha-1}}{\left[\beta \xi^{\alpha-1}-\Gamma(\alpha)\right] \Gamma(\alpha)} \int_{t_{1}}^{t_{2}} g(s, u(s)) d s \\
& +\frac{\beta\left(t_{2}^{\alpha-1}-t_{1}^{\alpha-1}\right)}{\left[\beta \xi^{\alpha-1}-\Gamma(\alpha)\right] \Gamma(\alpha)} \int_{t_{2}}^{1}(\xi-s)^{\alpha-1} g(s, u(s)) d s \\
& -\frac{\beta t_{1}^{\alpha-1}}{\left[\beta \xi^{\alpha-1}-\Gamma(\alpha)\right] \Gamma(\alpha)} \times \int_{t_{1}}^{t_{2}}(\xi-s)^{\alpha-1} g(s, u(s)) d s \mid \\
& \leq \frac{2 M}{\Gamma(\alpha+1)}\left(t_{2}-t_{1}\right)^{\alpha}+\frac{M \Gamma(\alpha)\left(t_{2}^{\alpha-1}-t_{1}^{\alpha-1}\right) t_{1}}{\left[\beta \xi^{\alpha-1}-\Gamma(\alpha)\right] \Gamma(\alpha)} \\
& +\frac{M \Gamma(\alpha) t_{2}^{\alpha-1}\left(t_{2}-t_{1}\right)}{\left[\beta \xi^{\alpha-1}-\Gamma(\alpha)\right] \Gamma(\alpha)} \\
& \begin{array}{l}
+\frac{M \beta\left(t_{2}^{\alpha-1}-t_{1}^{\alpha-1}\right) t_{1}}{\left[\beta \xi^{\alpha-1}-\Gamma(\alpha)\right] \Gamma(\alpha)}+\frac{M \beta t_{2}^{\alpha-1}\left(t_{2}-t_{1}\right)}{\left[\beta \xi^{\alpha-1}-\Gamma(\alpha)\right] \Gamma(\alpha)} \\
+\frac{M \Gamma(\alpha)\left(t_{2}^{\alpha-1}-t_{1}^{\alpha-1}\right)\left(1-t_{2}\right)}{\left[\beta \xi^{\alpha-1}-\Gamma(\alpha)\right] \Gamma(\alpha)}+\frac{M \Gamma(\alpha) t_{1}^{\alpha-1}\left(t_{2}-t_{1}\right)}{\left[\beta \xi^{\alpha-1}-\Gamma(\alpha)\right] \Gamma(\alpha)} \\
+\frac{M \beta\left(t_{2}^{\alpha-1}-t_{1}^{\alpha-1}\right)\left(1-t_{2}\right)}{\left[\beta \xi^{\alpha-1}-\Gamma(\alpha)\right] \Gamma(\alpha)}+\frac{M \beta t_{1}^{\alpha-1}\left(t_{2}-t_{1}\right)}{\left[\beta \xi^{\alpha-1}-\Gamma(\alpha)\right] \Gamma(\alpha)} .
\end{array} \\
& \left|T u\left(t_{1}\right)-T u\left(t_{2}\right)\right| \\
& \leq\left|\int_{0}^{1}\left[G\left(t_{2}, s\right)-G\left(t_{1}, s\right)\right] g(s, u(s)) d s\right| \\
& =\mid \int_{0}^{t_{2}} \frac{-\left(t_{2}-s\right)^{\alpha-1}}{\Gamma(\alpha)} g(s, u(s)) d s \\
& +\frac{1}{\left[\beta \xi^{\alpha-1}-\Gamma(\alpha)\right]} \int_{0}^{t_{2}} t_{2}^{\alpha-1} g(s, u(s)) d s \\
& +\frac{1}{\beta \xi^{\alpha-1}-\Gamma(\alpha)} \int_{t_{2}}^{1} t_{2}^{\alpha-1} g(s, u(s)) d s \\
& +\int_{0}^{t_{1}} \frac{-\left(t_{1}-s\right)^{\alpha-1}}{\Gamma(\alpha)} g(s, u(s)) d s \\
& -\frac{1}{\left[\beta \xi^{\alpha-1}-\Gamma(\alpha)\right]} \int_{0}^{t_{1}} t_{1}^{\alpha-1} g(s, u(s)) d s \\
& -\frac{1}{\beta \xi^{\alpha-1}-\Gamma(\alpha)} \int_{t_{1}}^{1} t_{1}^{\alpha-1} g(s, u(s)) d s \mid \\
& =\mid \int_{0}^{t_{1}}\left[\frac{-\left(t_{2}-s\right)^{\alpha-1}}{\Gamma(\alpha)}+\frac{\left(t_{1}-s\right)^{\alpha-1}}{\Gamma(\alpha)}\right] g(s, u(s)) d s \\
& +\int_{t_{1}}^{t_{2}} \frac{-\left(t_{2}-s\right)^{\alpha-1}}{\Gamma(\alpha)} g(s, u(s)) d s \\
& +\frac{\left(t_{2}^{\alpha-1}-t_{1}^{\alpha-1}\right)}{\beta \xi^{\alpha-1}-\Gamma(\alpha)} \int_{0}^{t_{1}} g(s, u(s)) d s \\
& +\frac{t_{2}^{\alpha-1}}{\beta \xi^{\alpha-1}-\Gamma(\alpha)} \int_{t_{1}}^{t_{2}} g(s, u(s)) d s \\
& +\frac{\left(t_{2}^{\alpha-1}-t_{1}^{\alpha-1}\right)}{\beta \xi^{\alpha-1}-\Gamma(\alpha)} \int_{t_{2}}^{1} g(s, u(s)) d s \\
& +\frac{t_{1}^{\alpha-1}}{\beta \xi^{\alpha-1}-\Gamma(\alpha)} \int_{t_{1}}^{t_{2}} g(s, u(s)) d s \mid \\
& \leq \frac{2 M}{\Gamma(\alpha+1)}\left(t_{2}-t_{1}\right)^{\alpha}+\frac{M\left(t_{2}^{\alpha-1}-t_{1}^{\alpha-1}\right) t_{1}}{\beta \xi^{\alpha-1}-\Gamma(\alpha)}
\end{aligned}
$$




$$
\begin{aligned}
& +\frac{M t_{2}^{\alpha-1}\left(t_{2}-t_{1}\right)}{\beta \xi^{\alpha-1}-\Gamma(\alpha)}+\frac{M\left(t_{2}^{\alpha-1}-t_{1}^{\alpha-1}\right)\left(1-t_{2}\right)}{\beta \xi^{\alpha-1}-\Gamma(\alpha)} \\
& +\frac{M t_{1}^{\alpha-1}\left(t_{2}-t_{1}\right)}{\beta \xi^{\alpha-1}-\Gamma(\alpha)} .
\end{aligned}
$$

Therefore, the operator $T$ is equicontinuous, and by ArzelaAscoli theorem, $T$ is a compact operator. Now, the Schauder fixed-point theorem [23] shows that the operator $T$ has a fixed-point theorem and so FBVP (32)-(33) has a solution. Finally, we will prove that FBVP (4)-(5) has a positive solution. Suppose that $u_{1}(t)$ is a solution of FBVP (32)-(33). Since the function $f$ is nondecreasing in $u$, we have

$$
f(t, \mu(t)) \leq g\left(t, u_{1}(t)\right) \leq f(t, \nu(t)), \quad t \in[0,1] .
$$

Assuming $X(t)=v(t)-u_{1}(t)$,

$$
\begin{gathered}
D^{\alpha} X(t) \geq f(t, \nu(t))-g\left(t, u_{1}(t)\right) \geq 0, \\
X(0)=X^{\prime}(0)=0, \quad D^{\alpha-1} X(1)=\beta X(\xi) .
\end{gathered}
$$

By Lemma 7, $X(t) \geq 0$; that is, $u_{1}(t) \leq v(t)$ for $t \in[0,1]$. Similarly, $\mu(t) \leq u_{1}(t)$ for $t \in[0,1]$. Therefore $u_{1}(t)$ is a positive solution of FBVP (4)-(5). The proof is complete.

Example 11. Consider the following fractional boundary value problem:

$$
\begin{gathered}
-D_{0^{+}}^{5 / 2} u(t)=f(t, u(t)), \quad 0<t<1, \\
u(0)=u^{\prime}(0)=0, \quad D_{0^{+}}^{\alpha-1} u(1)=\beta u(\xi),
\end{gathered}
$$

where

$$
f(t, u(t))=\sin \left(\frac{\pi t}{2}\right)+\sqrt{u}
$$

For $0 \leq k \leq 1$, we have $\sqrt{k} \leq 1$. Therefore

$$
\begin{aligned}
k^{1 / 2} f(t, u(t)) & =k^{1 / 2}\left(\sin \frac{\pi}{2} t+\sqrt{u}\right) \\
& \leq \sin \frac{\pi}{2} t+\sqrt{k u}=f(t, k u(t)) .
\end{aligned}
$$

Now, by Theorem 10, we obtain that the FBVP (39) has a positive solution.

\section{References}

[1] K. B. Oldham and J. Spanier, The Fractional Calculus, Academic Press, New York, NY, USA, 1974.

[2] B. Ross, Ed., The Fractional Calculus and Its Applications, vol. 475 of Lecture Notes in Mathematics, Springer, Berlin, Germany, 1975.

[3] F. B. Tatom, "The relationship between fractional calculus and fractals," Fractals, vol. 3, no. 1, pp. 217-229, 1995.

[4] T. F. Nonnenmacher and R. Metzler, "On the Riemann-Liouville fractional calculus and some recent applications," Fractals, vol. 3, no. 3, pp. 557-566, 1995.
[5] S. G. Samko, A. A. Kilbas, and O. I. Marichev, Fractional Integrals and Derivatives (Theory and Application), Gordon and Breach Science, Yverdon, Switzerland, 1993.

[6] A. A. Kilbas, H. M. Srivastava, and J. J. Trujillo, Theory and Applications of Fractional Differential Equations, vol. 204 of North-Holland Mathematics Studies, Elsevier Science B.V., Amsterdam, The Netherlands, 2006.

[7] K. S. Miller and B. Ross, An Introduction to the Fractional Calculus and Fractional Differential Equations, John Wiley \& Sons, New York, NY, USA, 1993.

[8] I. Podlubny, Fractional Differential Equations, vol. 198 of Mathematics in Science and Engineering, Academic Press, San Diego, Calif, USA, 1999.

[9] V. Lakshmikantham, S. Leela, and J. Vasundhara, Theory of Fractional Dynamic Systems, Cambridge Academic Publishers, Cambridge, UK, 2009.

[10] R. P. Agarwal, M. Benchohra, and B. A. Slimani, "Existence results for differential equations with fractional order and impulses," Memoirs on Differential Equations and Mathematical Physics, vol. 44, pp. 1-21, 2008.

[11] R. P. Agarwal, M. Benchohra, and S. Hamani, "Boundary value problems for fractional differential equations," Georgian Mathematical Journal, vol. 16, no. 3, pp. 401-411, 2009.

[12] C. Yu and G. Z. Gao, "On the solution of nonlinear fractional order differential equation," Nonlinear Analysis: Theory, Methods \& Applications, vol. 63, no. 5-7, pp. e971-e976, 1998.

[13] B. Ahmad and J. J. Nieto, "Existence results for a coupled system of nonlinear fractional differential equations with threepoint boundary conditions," Computers \& Mathematics with Applications, vol. 58, no. 9, pp. 1838-1843, 2009.

[14] A. M. Nahušev, “The Sturm-Liouville problem for a second order ordinary differential equation with fractional derivatives in the lower terms," Doklady Akademii Nauk SSSR, vol. 234, no. 2, pp. 308-311, 1977.

[15] S. Zhang, "Positive solutions for boundary-value problems of nonlinear fractional differential equations," Electronic Journal of Differential Equations, vol. 36, pp. 1-12, 2006.

[16] M. Benchohra, J. Henderson, S. K. Ntouyas, and A. Ouahab, "Existence results for fractional order functional differential equations with infinite delay," Journal of Mathematical Analysis and Applications, vol. 338, no. 2, pp. 1340-1350, 2008.

[17] D. Băleanu, K. Diethelm, E. Scalas, and J. J. Trujillo, Fractional Calculus Models and Numerical Methods, vol. 3 of Series on Complexity, Nonlinearity and Chaos, World Scientific, River Edge, NJ, USA, 2012.

[18] D. Băleanu and J. J. Trujillo, "On exact solutions of a class of fractional Euler-Lagrange equations," Nonlinear Dynamics, vol. 52, no. 4, pp. 331-335, 2008.

[19] D. Băleanu and O. G. Mustafa, "On the global existence of solutions to a class of fractional differential equations," Computers \& Mathematics with Applications, vol. 59, no. 5, pp. 1835-1841, 2010.

[20] M. El-Shahed and J. J. Nieto, "Nontrivial solutions for a nonlinear multi-point boundary value problem of fractional order," Computers \& Mathematics with Applications, vol. 59, no. 11, pp. 3438-3443, 2010.

[21] M. El-Shahed, "Positive solutions for boundary value problem of nonlinear fractional differential equation," Abstract and Applied Analysis, vol. 2007, Article ID 10368, 8 pages, 2007. 
[22] S. Liang and J. Zhang, "Positive solutions for boundary value problems of nonlinear fractional differential equation," Nonlinear Analysis: Theory, Methods \& Applications, vol. 71, no. 11, pp. 5545-5550, 2009.

[23] D. Guo and J. Zhang, Nonlinear Fractional Analysis, Science and Technology Press, Jinan, China, 1985. 


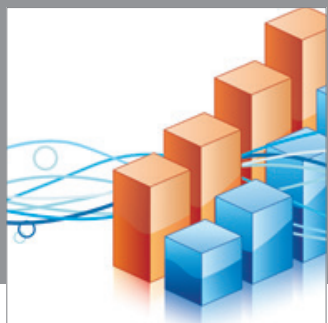

Advances in

Operations Research

mansans

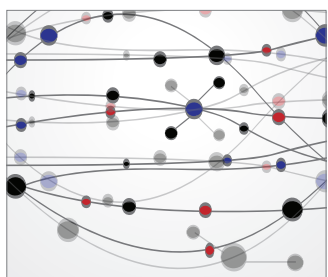

The Scientific World Journal
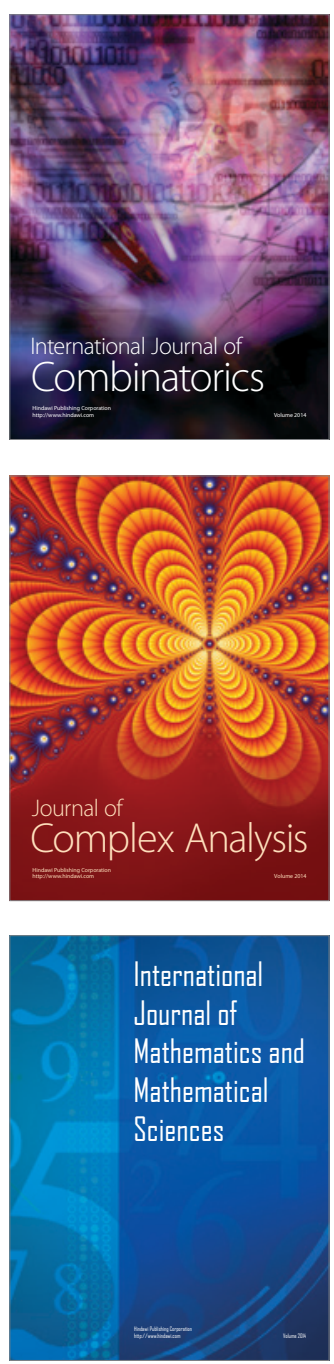
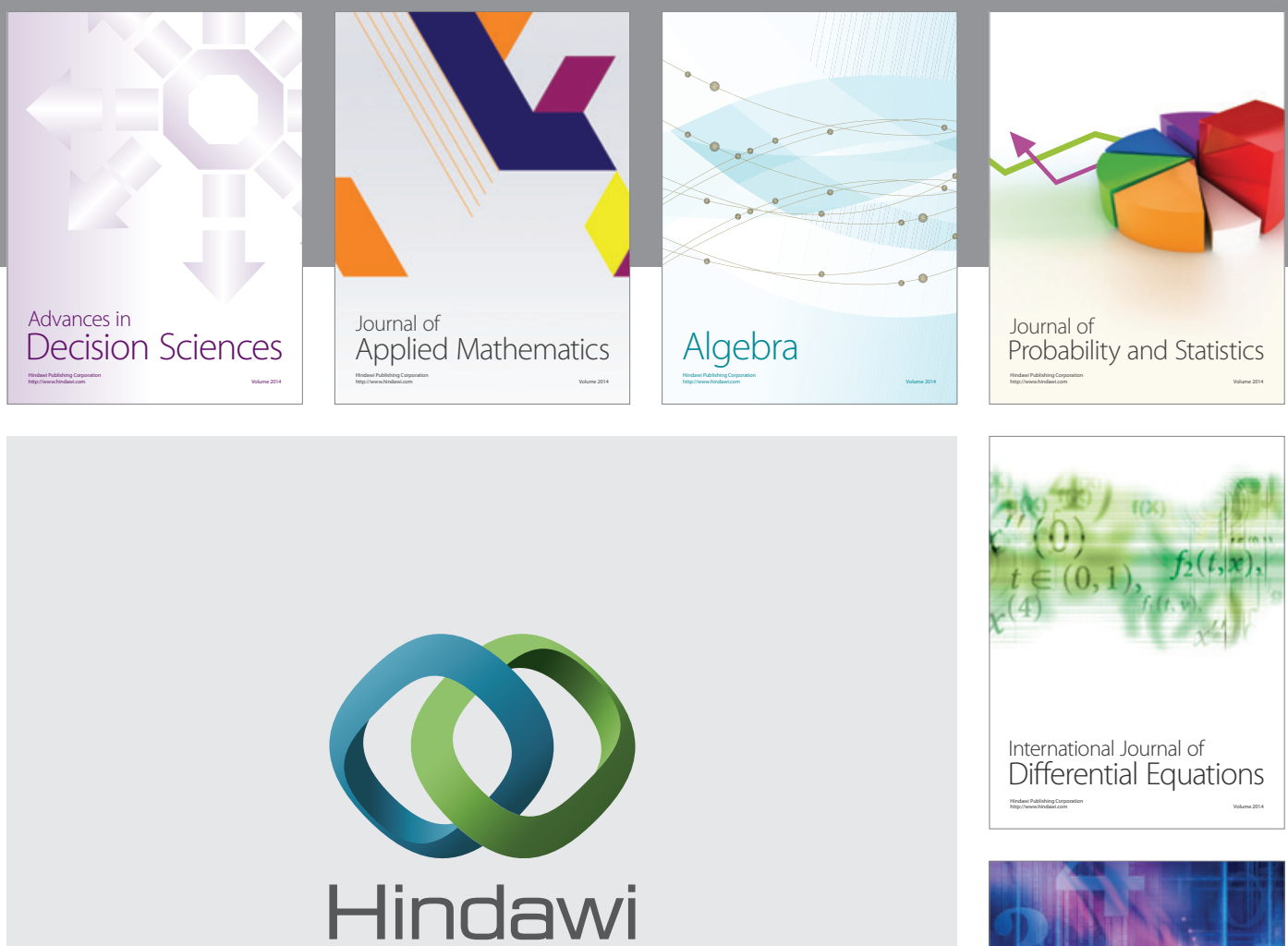

Submit your manuscripts at http://www.hindawi.com
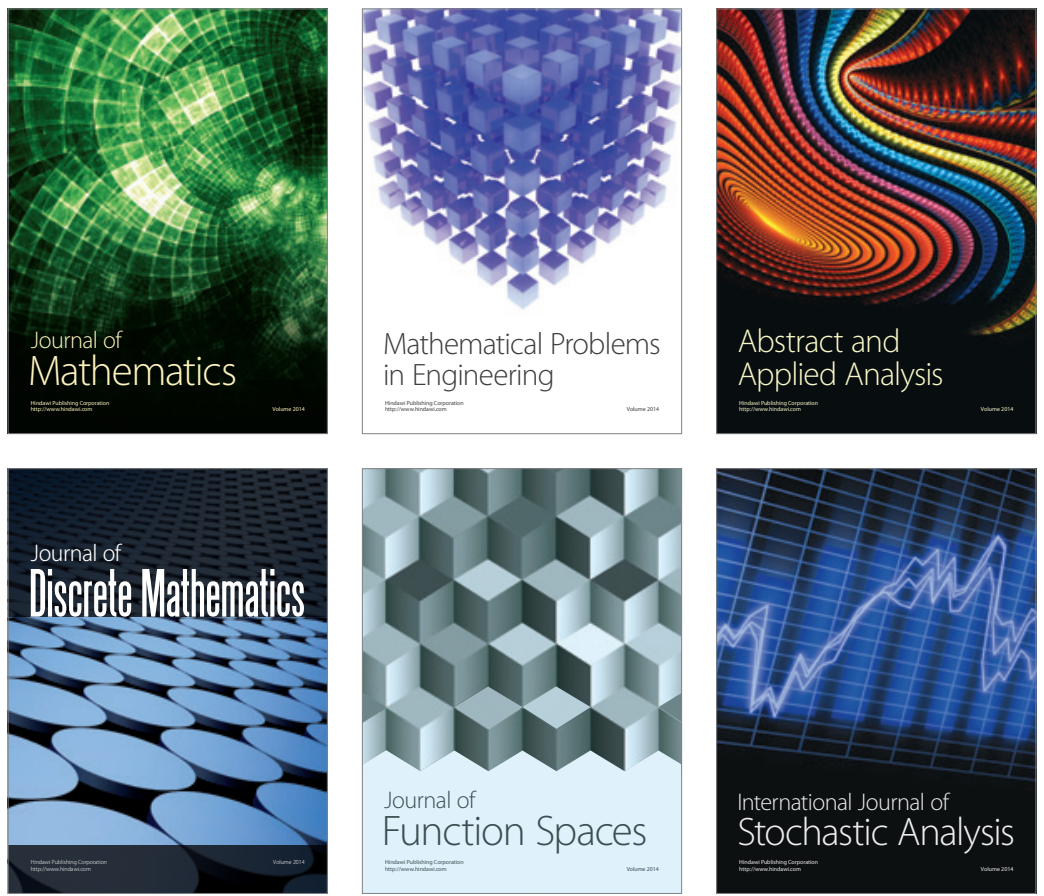

Journal of

Function Spaces

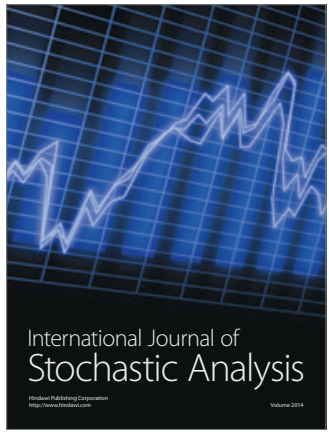

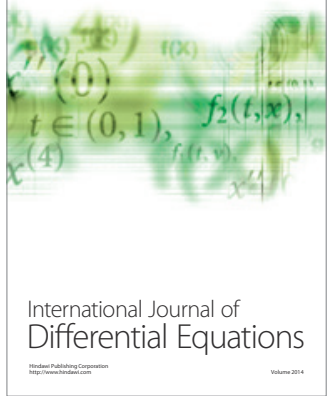
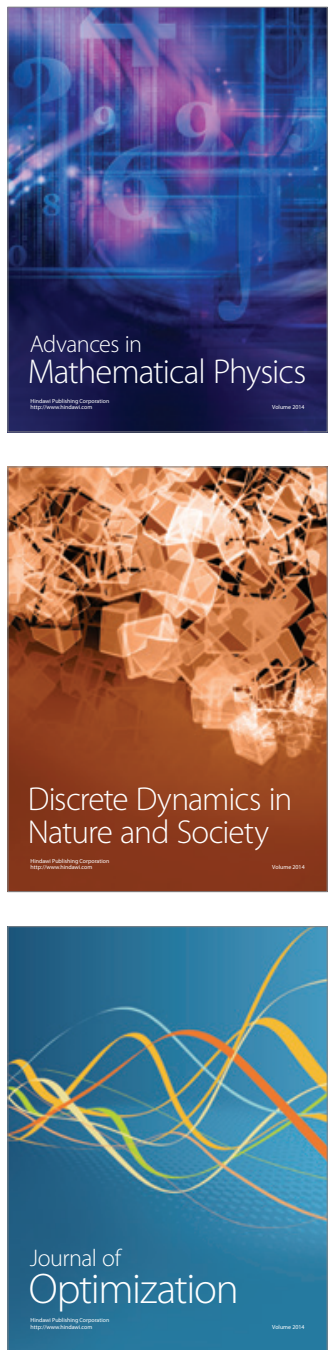